\title{
Prosthetic mechanobiology - how using a prosthesis can lead to the development of Deep Tissue Injuries
}

Marisa Graser ${ }^{1}$, Sarah Day ${ }^{1}$, Arjan Buis ${ }^{1}$

${ }^{1}$ University of Strathclyde

\section{BACKGROUND (70 words)}

Deep Tissue Injury (DTI) is a form of pressure ulcer that forms when soft tissues experience prolonged or high pressure and shear. Our understanding of this condition increased significantly over the last two decades, with major research on immobilised individuals. However, transtibial prosthetic users might also be at risk of developing DTI: Following amputation, the soft tissues of the residuum become part of the weight-bearing system, despite being inapt to withstand high loads.

\section{AIM (30 words)}

To understand DTI development in prosthetic users and identify gaps in existing research, we carried out a scoping review with a focus on population-specific aetiology, risk factors and methodologies to investigate both.

\section{METHOD (100 words)}

The scoping review followed a methodological framework by the Joanna Briggs Institiute [1], in compliance with the PRISMA-ScR checklist for scoping reviews [2]. Qualitative data was collected based on keywords related to transtibial prosthetic use in combination with DTI development. The database search in Pubmed, Ovid Excerpta Medica, and Scopus, together with reference lists and forward-citations returned 16 peer reviewed, English language studies.

\section{RESULTS (130 words)}

We addressed three key areas: Firstly, the aetiology of DTI is a complex interplay between cellular deformation and the impairment of lymphatic and microvascular systems. However, the influence of prosthesis-specific aspects like dynamic loading and amputation-related alterations of the ancillary systems remain unclear.

Secondly, we found several risk factors that make transtibial prosthetic users susceptible to DTI. Intrinsic determinants are amputation-related changes of the tissue morphology and its mechanical properties, and sensory impairment of the residuum. Extrinsic factors are the socket design and choice of prosthetic components.

Finally, most studies used a methodology based on biomechanics. They commonly combined different loading conditions with medical imaging and computational analysis. However, we found a great variety of input data, modelling assumptions, and outcome measures. Additionally, the variability in geometrical patient characteristics and prosthetic componentry was high.

\section{DISCUSSION AND CONCLUSION (70 words)}

The results indicate that transtibial prosthetic users may be at risk of DTI development. This is dependent on a complex interplay of mechanical, anatomical, and physiological factors. However, fundamental research on cell and tissue reactions to dynamic loading and on the ancillary system is missing. Additionally, high inter-patient variability and methodological limitations complicate the interpretation of outcome measures. We therefore recommend increased interdisciplinary research to widen our understanding of DTI in transtibial prosthetic users, which has the potential to initiate muchneeded advances in surgical, and prosthetic practice.

\section{REFERENCES}

1. Peters M; 2017, Joanna Briggs Institute Reviewer's Manual [Online]

2. Tricco AC; 2018, Ann Intern Med. 